\title{
PAD: A Pancreatic Cancer Detection based on Extracted Medical Data through Ensemble Methods in Machine Learning
}

\author{
Santosh Reddy $\mathrm{P}^{* 1}$ \\ Research Scholar \\ Department of CSE \\ Presidency University, Bengaluru
}

\author{
Chandrasekar $\mathrm{M}^{2}$ \\ Associate Professor \\ Department of CSE \\ Presidency University, Bengaluru
}

\begin{abstract}
The considerable research into medical health systems is allowing computing systems to develop with the most cutting-edge innovations. These developments are paving the way for more efficient medical system implementations, including automatic identification of health-related disorders. The most important health research is being done to predict cancer, which can take several forms and affect many parts of the body. One of the most prevalent tumors that is expected to be incurable is pancreatic cancer. Pancreatic cancer is one of the most common cancers that is projected to be incurable. Previous research has found that a panel of three protein biomarkers (LYVE1, REG1A, and TFF1) found in urine can help detect respectable PDAC. To improve this panel in this study by replacing REG1A with REG1B from extracted data sets into CSV format. Finally, will analyze four significant biomarkers that are found in urine, creatinine, LYVE1, REG1B, and TFF1. Creatinine is a protein that is commonly utilized as a kidney function indicator. Lymphatic vessel endothelial hyaluronan receptor 1 (YVLE1) is a protein that may help tumors spread. REG1B is a protein that has been linked to pancreatic regeneration, while TFF1 is trefoil factor 1 , which has been linked to urinary tract regeneration and repair It's impossible to treat it properly once it's been diagnosed. Machine learning and neural networks are now showing promise for accurate pancreatic picture segmentation in real time for early diagnosis. This research looks at how to analyze pancreatic tumors using ensemble approaches in machine learning. According to preliminary data, the proposed technique looks to improve the classifier's performance for early diagnosis of pancreatic cancer.
\end{abstract} CA19_9

Keywords-Pancreatic; PDAC; LYVE1; REG1A; TFF1;

\section{INTRODUCTION}

Cancer, according to medical health news analysis, is one of the most troublesome diseases that can appear to be invincible at times. It's possible that it's a hereditary disease because it's caused by abnormalities in genes that control how cells in the human body work. These genetic alterations might be passed down through generations or caused by a person's lifestyle. It is an important organ of the human body, has internal and external secretory functions and is disposed to various diseases. Surgical right of entry and for which prebiopsy is repeatedly impossible [1-5]. Pancreatic cancer is the fourth majority common source of cancer death and the second most important cause of death from neoplasm's disturbing the digestive coordination.

However, regular segmentation of the pancreas remainders a dispute for the subsequent reasons: 1) low soft tissue contrast on CT images. 2) Huge anatomical variations. The pancreas shows great anatomical unpredictability in terms of size and location in the abdominal cavity of patients [6][7]. the pancreas is a deformable yielding tissue. Consequently, the outline and manifestation of the pancreas have great differences in dissimilar individuals. PDAC (pancreatic ductal adenocarcinoma) is a particularly lethal form of pancreatic cancer. The five-year survival rate is less than $10 \%$ once diagnosed. However, if the cancer is caught early enough, when tumours are still small and manageable, 5-year survival rates can reach $70 \%$. Unfortunately, many cases of pancreatic cancer go undetected until the disease has progressed throughout the body. As a result, a diagnostic test to detect pancreatic cancer patients could be quite beneficial. Traditionally, blood has been the primary source of biomarkers, however urine is a viable alternative biological fluid. It enables non-invasive sample, high-volume collection, and repeated measurements with ease. There are currently no reliable biomarkers for detecting PDAC earlier. Serum CA199 , the only biomarker utilized in clinical practice, is not specific or sensitive enough for screening and is primarily employed as a prognostic marker and for monitoring treatment response.

Even though to collects invasive samples, he increases cancer diagnosis when combined with other urine indicators in a study. Previous research has found that a panel of three protein biomarkers (LYVE1, REG1A, and TFF1) found in urine can help detect significant PDAC. We improved this panel in this study by replacing REG1A with REG1B. Finally, we will analyze four significant biomarkers that are found in urine: creatinine, LYVE1, REG1B, and TFF1. Creatinine is a protein that is commonly utilized as a kidney function indicator. Lymphatic vessel endothelial hyaluronan receptor 1 (YVLE1) is a protein that may help tumors spread. REG1B is a protein that has been linked to pancreatic regeneration, while TFF1 is trefoil factor 1 , which has been linked to urinary tract regeneration and repair.

*Corresponding Author 


\section{LITERATURE SURVEY}

The concept of regular automation algorithms and suggest that Support Vector Machine (SVM) is an authoritative classification process for classifying data related to the calculation of Wisconsin Breast Cancer data with a minor proportion of time [8]. Proportion of relative results in stipulations of effectiveness and effectiveness of four algorithms of differences in data retrieval and automatic automation. Initiates a new functioning favor of the medical health system with the intention of predicts the outcome of an average patient in the examination of electronic medical proceedings and the recognized parameters of parameters established for proper functioning [9]. The efficient prognostic data is normally provided by the application coordination with the estimate of data for variable effects, types of effects and the threshold parameter to identify the diagnosis of the disease. Corresponding the medical proceedings, they use and cover for blood cancer, heart failure, diabetes [10].

To develop a function based on the red convolution neuronal representation to analyze rectal prescribed amount sharing and predict rectal toxicity in patients with uterine cancer, by means of data as of combined radiotherapy (EBRT) and brachytherapy (BT) [11]. They adopted is a somewhere to live and transfer strategy to influence patient data. The adaptive synthetic model technique is used to increase the dates for footage data losses and loss factors. Produce Gradient Activation Weight Map (Grad-CAM) classes to generate RSDM discriminate regions with the calculate model. The $\mathrm{CNN}$-based representation for predicting rectal dose by means of transfer therapy for uterine cancer radiotherapy is analyzed by means of a conjunction of experimental outcome [12].

\section{METHODS AND MATERIALS}

Neural Designer was used to tackle this problem. You can utilise the trial to follow it step by step. Because the variable to be predicted is categorical, this is a classification project (no pancreatic disease, benign hepatobiliary disease, or pancreatic cancer). The goal is predicting the presence of disease before it's diagnosed, and more specifically, differentiating between pancreatic cancer versus non-cancerous pancreas condition and healthy condition

\section{A. Data Set}

Barts Pancreas Tissue Bank, University College London, University of Liverpool, Spanish National Cancer Research Centre, Cambridge University Hospital, and University of Belgrade all contributed to the data collection. A total of 590 urine samples were tested for the biomarker panel, including 183 control samples, 208 benign hepatobiliary disease samples (of which 119 were chronic pancreatitis), and 199 PDAC samples. Data source, Variables, Instances, and Missing values are the four concepts that make up this system. The information used to generate the model is contained in the data file pancreatic-cancer.csv. There are 509 rows and 14 columns in all. The rows represent the study samples, while the columns representvarious cancer risk variables.

This data collection makes use of the following 16 variables: id of the sample. Each subject is identified by a unique string called a cohort. Cohort 1 samples has been used previously. Cohort 2 samples have been added, with the following sample origin: BPTB: Barts Pancreas Tissue Bank, London, UK; ESP: Spanish National Cancer Research Centre, Madrid, Spain; LIV: Liverpool University, UK; UCL: University College London, UK; BPTB: Barts Pancreas Tissue Bank, London, UK; BPTB: Barts Pancreas Tissue Bank, London, UK; CA 19-9 monoclonal antibody levels in blood plasma, which are frequently elevated in pancreatic cancer patients. Only 350 participants were analysed (one goal of the study was to compare different CA 19-9 cut points from a blood sample to a model built using urine samples), creatinine: A urinary biomarker of renal function. LYVE1: Lymphatic vessel endothelial hyaluronan receptor 1 is a protein discovered in the urine that may have a role in tumour spread. REG1A: Urinary levels of a protein connected to pancreatic regeneration, REG1B: Urinary levels of a protein linked to pancreatic regeneration, REG1B: Urinary levels of a protein linked to pancreatic regeneration TFF1: Only 306 patients had their urinary Trefoil Factor 1 levels evaluated, which could be linked to urinary tract regeneration and repair (one purpose of the study was to assess REG1B vs. REG1A). 3 = Pancreatic ductal adenocarcinoma; 2 = benign hepatobiliary disease (119 of which are chronic pancreatitis) i.e., pancreatic cancer, benign sample diagnosis: Stage: For those who have been diagnosed with a benign, non-cancerous condition, stage: IA, IB, IIA, IIIB, III, IV are the stages of pancreatic cancer. There are a few input variables that must be marked as unused among all of them. Specifically, 'sample id', which Neural Designer does automatically, 'sample origin', which only specifies the origin of the patient samples and should not affect the final diagnosis, 'stage,' which is a variable that only exists for people we already know have cancer, 'patient cohort,' which does not contribute to the final sample diagnosis, and 'benign sample diagnosis,' which is a variable that does not contribute to the final sample diagnosis. The variable corresponding to the biomarker REG1A is not in all the samples of the study. For that reason, we choose to set it as unused too. This decision will not mean a deterioration of the model as the biomarker REG1B improve the results. Once the data set is configured, we can calculate the data distribution of the variables. The following figure depicts the number of patients who have cancer and those who do not. The minimum frequency is $31.0169 \%$, which corresponds to no pancreatic disease diagnosis. The maximum frequency is $35.2542 \%$, which corresponds to benign hepatobiliary disease diagnosis. As we can see, all the samples are well distributed between the three cases.

There should be a partition our dataset into four subsets to compare the accuracy and AUC (Area Under Curve) calculated in this study with those in the paper listed in the references section. Control samples vs. PDAC stages I and II: We only chose healthy person samples and pancreatic cancer stages I and II samples from the raw dataset. Control samples vs. PDAC stages III and IV: Only healthy individual samples and pancreatic cancer stages III and IV samples were chosen from the raw dataset. Benign hepatobiliary disorders vs. PDAC stages I and II: We selected individuals with benign tumour samples and pancreatitis cancer stages I and II samples from the raw dataset. PDAC stages III and IV vs. benign hepatobiliary diseases: Only individuals with benign tumour samples and 
pancreatic cancer stages I and II are chosen from the raw dataset. In all these scenarios, the examples are separated into training and testing subsets, with each subset having half of the samples.

\section{B. Stage I \& Stage II with Sample Data}

The inputs-target correlations of all the inputs with the target are shown in the Fig. 1. This allows us to see how different inputs affect the default.

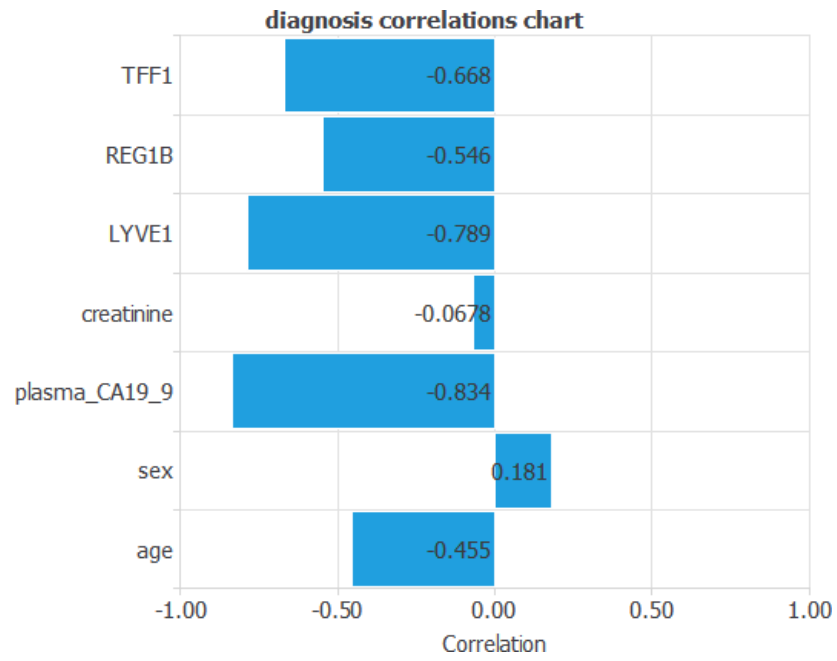

Fig. 1. Diagnosis Correlation Chart for Stage $1 \& 2$.

The biomarkers LYVE1 and plasma CA19 9 are the most highly associated variables.

\section{Stage III \& Stage IV with Sample Data}

Fig. 2 shows inputs-target correlations of all the inputs with the biomarkers LYVE1 and TFF1 are the most highly associated variables.

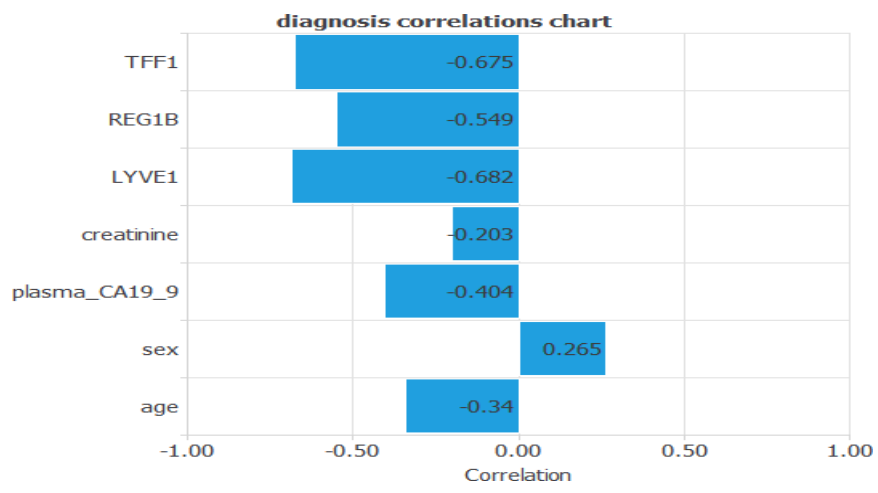

Fig. 2. Diagnosis Correlation Chart for Stage $3 \& 4$.

\section{Implementation}

The above Fig. 3 shows the system Design. IPancreatic cancer is one of the most devastating types of cancer, with something like a terrible prognosis in the present environment. Because of its complex visual appearance and indistinct curvature, the pancreas border line is difficult to distinguish from its anatomies in CT/MRI scans. Most relevant health research is available on cancer prediction, which comes in a variety of forms and can affect different sections of the body. Pancreatic cancer is one of the most common cancers that is projected to be incurable. Once diagnosed, it cannot be treated adequately. Machine learning and neural networks are providing promising findings for accurate pancreatic picture segmentation in real time early detection these days. Pancreatic cancer can be classified into five stages. The size and location of the tumour, as well as whether the cancer has spread to the liver, lungs, or abdominal cavity, will determine your diagnosis. It's possible that it's spread to nearby organs, tissues, or lymph nodes. Make sure to discuss your case with your healthcare practitioner. Understanding your pancreatic cancer prognosis might assist you in making an informed treatment selection. According to previous studies, a panel of three protein biomarkers present in urine (LYVE1, REG1A, and TFF1) can assist detect significant PDAC.

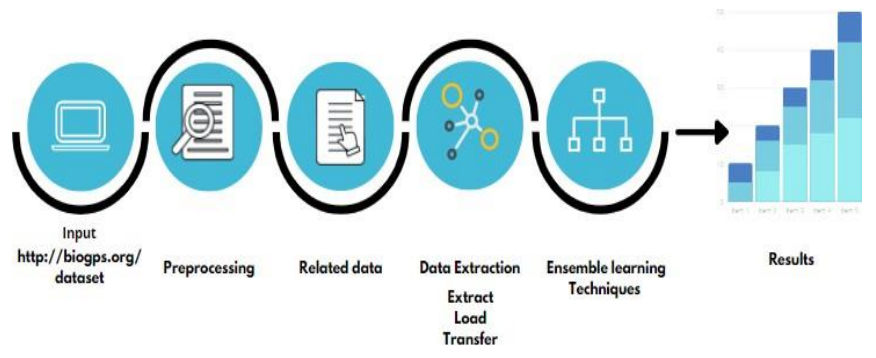

Fig. 3. System Design.

We improved this panel in this study by replacing REG1A with REG1B. Finally, we will analyse four significant biomarkers that are found in urine: creatinine, LYVE1, REG1B, and TFF1. Creatinine is a protein that is commonly utilised as a kidney function indicator. Lymphatic vessel endothelial hyaluronan receptor 1 (YVLE1) is a protein that may help tumours spread. REG1B is a protein that has been linked to pancreatic regeneration, while TFF1 is trefoil factor 1 , which has been linked to urinary tract regeneration and repair It's impossible to treat it properly once it's been diagnosed. Machine learning and neural networks are now showing promise for accurate pancreatic picture segmentation in real time for early diagnosis.

1) Naive bayes: To make it easier to understand, I'll go over the theory behind Naive Bayes first, and then use an example to clarify the notions. The Bayes Theorem, which asserts the following equation, inspired the Naive Bayes Classifier.

$P(A \mid B)=\frac{P(B \mid A) * P(A)}{P(B)}$

Rewrite the equation using $\mathrm{X}$ (input variables) and $\mathrm{y}$ (output variables) to make it easier to understand (output variable). In plain English, this equation calculates the probability of $y$ given input attributes $X$.

$P(y \mid X)=\frac{P(X \mid y) * P(y)}{P(X)}$

We may rewrite $\mathrm{P}(\mathrm{X} \mid \mathrm{y})$ as follows because of the naive assumption (therefore the name) that variables are independent given the class.

$\mathrm{P}(\mathrm{X} \mid \mathrm{y})=\mathrm{P}\left(X_{1} \mid \mathrm{y}\right) * \mathrm{P}\left(X_{2} \mid \mathrm{y}\right) * \ldots \ldots \ldots * \mathrm{P}\left(X_{n} \mid \mathrm{y}\right)$ 
Also, because we're solving for $\mathrm{y}, \mathrm{P}(\mathrm{X})$ is a constant, so we can drop it from the equation and replace it with a proportionality. As a result, we arrive to the following equation.

$$
P(y \mid X) \propto P(X \mid y) * P(y)
$$

$\mathrm{Or}$

$P(y \mid X) \propto P(y) * \pi_{i=1}^{n} P\left(x_{i} \mid y\right)$

The purpose of Naive Bayes is to choose the classy with the highest probability now that we've reached at this equation. Argmax is a simple operation that finds the argument that gives the target function's maximum value. In this situation, we're looking for the highest y value.

2) Bagging \& boosting: When estimating a numerical outcome, aggregating, and voting with a plurality when predicting a class, BAGGING (Fig. 4) is the process of applying Bootstrap sampling on the training dataset, aggregating when estimating a numerical outcome, and voting with a plurality when predicting a class. Bagging, on the other hand, would degrade the performance of stable algorithms such as k-nearest neighbours discriminant analysis, and Nave bayes, because this algorithm uses initial samples that contain about 63 percent of the original data, meaning that each sample is missing about 37 percent of the original data. The Boosting strategy works by combining numerous simple learning algorithms instead of employing a very accurate prediction rule. The update approach then combines all these weak rules to reduce variations and deviations in the individual model rules, leading to a single prediction rule that is significantly more accurate than any of the weak rules alone. There are two main techniques for effectively applying the reinforcement algorithm.

Test error is the minuscule proportion of errors on a recently sampled test set. CT scans can be used to detect if cancer is present and has spread, as well as to guide a biopsy, and can be used to diagnose pancreatic cancer utilizing a variety of imaging modalities. MRIs are used when CT scans aren't a possibility or other tests aren't conclusive. An endoscope can be used to perform ultrasounds from outside the abdomen or through the digestive tract. Why is it so common for pancreatic cancer to be found so late? Because the pancreas is placed deep within the abdomen, it is difficult to identify early.

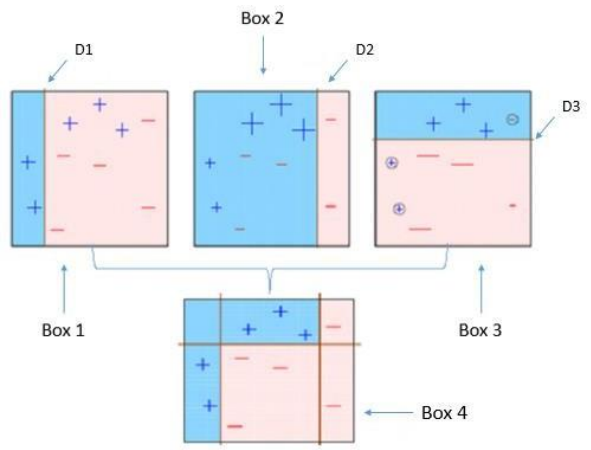

Fig. 4. Bagging \& Boosting.
For this stage of the model generation, we'll utilize the same neural network configuration for all four situations. Layers, Perceptron, and layers are used to solve classification problems. We recognize that having a perceptron layer adds to the neural network being overfit. As a result, the perceptron layer is removed. Let's start with bagging techniques. The following equation demonstrates the principle of bagging, which is short for bootstrap aggregation: On a bootstrapped dataset, train several weak learners $\mathrm{b}(\mathrm{x})$ and take the average to get the learning outcome. The term "bootstrap" refers to the process of producing different data samples from the original dataset at random (roll $\mathrm{n}$-faces dice $\mathrm{n}$ times).

$f(x)=\frac{1}{B} \sum_{b=1}^{B} f_{b}(x)$

Following this logic, the Random Forest algorithm is naturally introduced, as decision trees are an excellent option for weak learners. Low bias and large variance are two features of a single decision tree. Bias remains after aggregating a group of trees, although variance decreases. By developing a large enough random forest, we could attain a constant bias that is as low as possible.

Let's move on to boosting now. The main principle behind boosting is to see if a poor learner can be made to improve by focusing on their weaknesses. This is accomplished by repeatedly employing the weak learning method to generate a series of hypotheses, each one focused on the cases that the prior hypotheses found problematic and misclassified.

$f(x)=\sum_{t} \propto_{t} h_{t}(x)$

\section{E. Experimental Results}

1) Testing analysis with similarity index: The performance of the trained neural network is subsequently evaluated utilizing an extensive testing analysis. The conventional way is to compare the neural network's outputs against previously unseen data, known as testing instances. The ROC curve is a well-known method for evaluating generalization performance. This is a visual aid for studying the discrimination capabilities of the classifier. One of the parameters acquired from this graph is the area under the curve (AUC). The closer the classifier is to 1 area under the curve, the better.

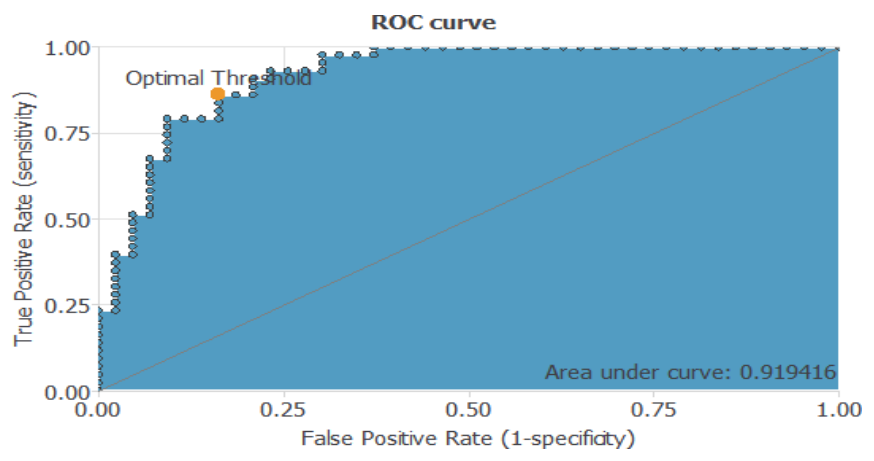

Fig. 5. ROC Curve for Control Samples \& PDAC Stage $1 \& 2$. 
a) Control Samples and PDAC Stage I and II: The AUC assumes a high value in this case: $\mathrm{AUC}=0.919$. The ideal threshold is calculated by identifying the point on the ROC curve (Fig. 5) that is closest to the upper left corner in Neural Designer. The ideal threshold is the one that corresponds to that point, and it has a value of 0.788 in this example. The confusion matrix and binary classification tests provide useful information regarding the performance of our predictive model. Both are shown below for their best choice threshold (Table I).

TABLE I. Predictive of Positive \& Negative Threshold Stage $1 \&$ STAGE 2

\begin{tabular}{|l|l|l|}
\hline & Predictive Positive & Predictive Negative \\
\hline Real Positive & $34(39.5 \%)$ & $6(7.0 \%)$ \\
\hline Real Negative & $9(10.5 \%)$ & $37(43.0 \%)$ \\
\hline
\end{tabular}

Classification accuracy: 82.6 percent (Ratio of correctly classified samples), Error rate: 17.4 percent (Ratio of misclassified samples), Sensitivity: $79.1 \%$ (Proportion of true positive samples that are projected positive), and Specificity: 86.0 percent (Portion of real negative predicted negative). The classification accuracy is good $(82.6 \%)$, indicating that the prediction is applicable to a broad number of scenarios.

b) Control Samples and PDAC Stage III and IV: The AUC takes a high value in this case: The ideal threshold is 0.587, and the AUC is 0.913 (Fig. 6 and Table II).

Classification accuracy: $88.6 \%$ (Ratio of correctly categorized samples), Error rate: 11.4 percent (Ratio of misclassified samples), Sensitivity: 92.4 percent (Percentage of genuine positive samples that are predicted positive), and Specificity: 81.3 percent (Portion of real negative predicted negative). The classification accuracy is good (88.6\%), indicating that the forecast is applicable to a vast number of scenarios.

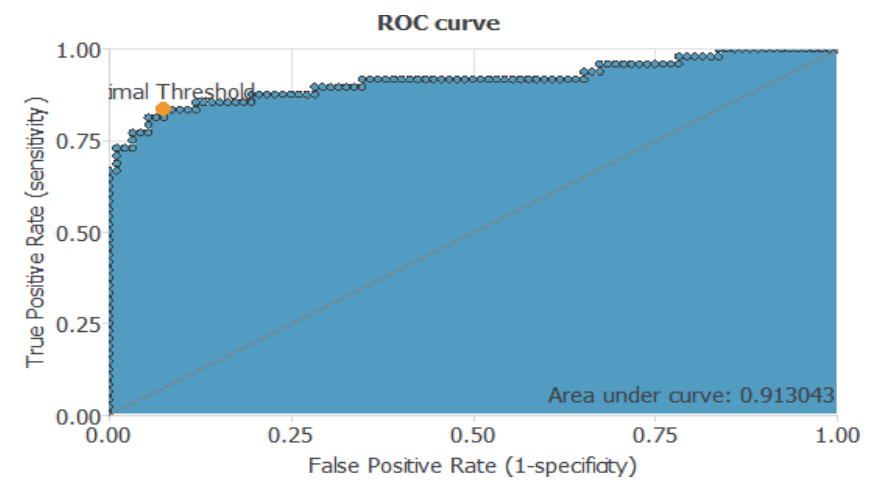

Fig. 6. ROC Curve for Control Samples \& PDAC Stage $3 \& 4$.

TABle II. Predictive of Positive \& Negative Threshold Stage 3 \& STAGE 4

\begin{tabular}{|l|l|l|}
\hline & Predictive Positive & Predictive Negative \\
\hline Real Positive & $85(60.7 \%)$ & $9(6.4 \%)$ \\
\hline Real Negative & $7(5.0 \%)$ & $39(27.9 \%)$ \\
\hline
\end{tabular}

c) Difference between Benign Hepatobiliary diseases and PDAC Stage I and II: The AUC takes a high value in this case: The ideal threshold is 0.653 , and the AUC is 0.920 (Fig. 7 \& Table III).

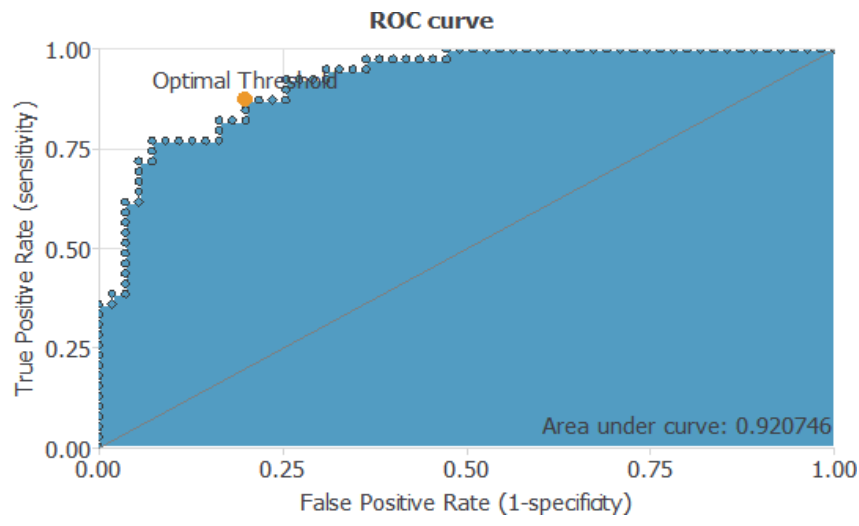

Fig. 7. ROC Curve for Benign Hepatobiliary Diseases \& PDAC Stage 1 $\& 2$.

TABLE III. PREDICTIVE OF POSITIVE \& NEGATIVE THRESHOLD BENIGN HePatobiliary Stage $1 \&$ Stage 2

\begin{tabular}{|l|l|l|}
\hline & Predictive Positive & Predictive Negative \\
\hline Real Positive & $44(46.8 \%)$ & $5(5.3 \%)$ \\
\hline Real Negative & $11(11.7 \%)$ & $34(36.2 \%)$ \\
\hline
\end{tabular}

Classification accuracy: $83.0 \%$ (Ratio of correctly classified samples), Error rate: $17.0 \%$ (Ratio of misclassified samples), Sensitivity: 80.0 percent (Proportion of true positive samples that are predicted positive), and Specificity: 87.2 percent (Portion of real negative predicted negative). The classification accuracy is good $(83.0 \%)$, indicating that the forecast is applicable to a vast number of scenarios.

d) Difference between Benign Hepatobiliary Disease and Stage III and Stage IV: The AUC takes a high value in this case: The ideal threshold is 0.412 , and the AUC is 0.848 \& (Table IV).

TABLE IV. Predictive OF Positive \& Negative Threshold BenigN HePatobiliary STAGE 3 \& Stage 4

\begin{tabular}{|l|l|l|}
\hline & Predictive Positive & Predictive Negative \\
\hline Real Positive & $47(52.8 \%)$ & $11(12.4 \%)$ \\
\hline Real Negative & $8(9.0 \%)$ & $23(25.28 \%)$ \\
\hline
\end{tabular}

Classification accuracy: $78.7 \%$ (Ratio of correctly classified samples), Error rate: 21.3 percent (Ratio of misclassified samples), Sensitivity: 85.5 percent (Percentage of true positive samples that are projected positive), and Specificity: $67.6 \%$ (Portion of real negative predicted negative). The classification accuracy is good $(78.7 \%)$, indicating that the forecast is appropriate in many circumstances. We'll show a table with some sensitivity and specificity cut-offs, just like in the paper. Table V, will look at the control samples vs. pancreatic cancer stages I and II, as well as stages III and IV: 
TABLE V. CONTROL SAMPLES vs PANCREATIC CANCER StAgE $1 \& 2$

\begin{tabular}{|l|l|l|}
\hline Sensitivity Cut-off & $\begin{array}{l}\text { Specificity (Controls } \\
\text { vs I, II) }\end{array}$ & $\begin{array}{l}\text { Specificity (Controls } \\
\text { vs III, IV) }\end{array}$ \\
\hline 0.8 & 0.86 & 0.875 \\
\hline 0.85 & 0.791 & 0.854 \\
\hline 0.9 & 0.744 & 0.833 \\
\hline 0.95 & 0.512 & 0.771 \\
\hline
\end{tabular}

Now Table VI will look at how benign samples compare to pancreatic cancer stages I and II, as well as stages III and IV:

TABLE VI. Benign SAmples vs Pancreatic CANCER Stage $3 \& 4$

\begin{tabular}{|l|l|l|}
\hline Sensitivity Cut-off & $\begin{array}{l}\text { Specificity (benign vs } \\
\text { I, II) }\end{array}$ & $\begin{array}{l}\text { Specificity (benign vs } \\
\text { III, IV) }\end{array}$ \\
\hline 0.8 & 0.846 & 0.676 \\
\hline 0.85 & 0.769 & 0.647 \\
\hline 0.9 & 0.769 & 0.618 \\
\hline 0.95 & 0.615 & 0.559 \\
\hline
\end{tabular}

e) Deployment of the Model: The neural network can be preserved for future usage in the so-called model deployment mode once its generalization performance has been evaluated. Calculating outputs, which generates a set of outputs for each set of inputs given, is an interesting activity in the model deployment tool. The outputs, in turn, are determined by the parameter values. Fig. 8 then, for the benign tumour or PDAC stages III and IV diagnosis, will offer an example. LYVE1: 3.78856, REG1B: 121.787, TFF1: 752.305, diagnosis: 0.6895, age: 45, sex: F (1), plasma CA19-9: 740.94, creatinine: 0.927814, LYVE1: 3.78856, REG1B:121.787, TFF1:752.305, diagnosis: 0.6895 That person's chance of pancreatic cancer (stages III or IV) would be high. Table VII and Table VIII shows the Model \& Detailed Accuracy by Class.

Fig. 9 shows the Detailed Accuracy by Class and Fig. 10 Shows the Association between CCI (Correctly classified Instances) and ICUI (Incorrectly class Unknown Instances). Fig. 11 shows the association between CCI, ICCI, ICUI, and TNI.

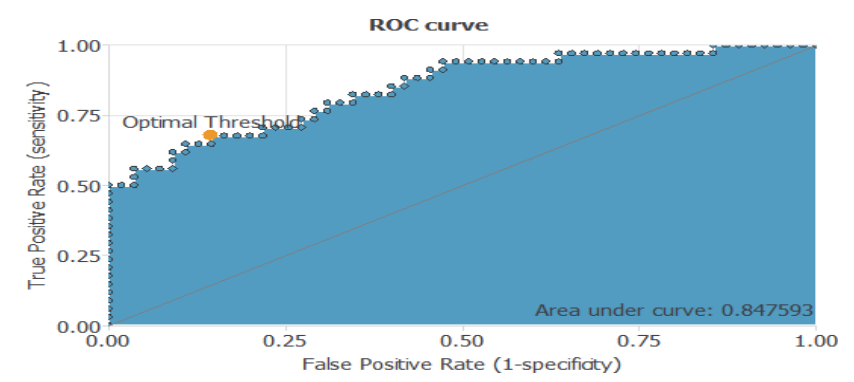

Fig. 8. ROC Curve for Benign Hepatobiliary diseases \& PDAC Stage $3 \& 4$.

TABLE VII. MODEL ACCURACY

\begin{tabular}{|c|c|c|c|c|c|c|c|c|c|c|c|}
\hline Algorithms & $\begin{array}{l}\text { Instance of } \\
\text { CCI }\end{array}$ & CCI & $\begin{array}{l}\text { Instance of } \\
\text { ICCI }\end{array}$ & ICCI & KS & MAE & RMSE & RAE & RRSE & ICUI & TNI \\
\hline Navie Bayes & 70 & $35.17 \%$ & 129 & $64.8 \%$ & 0.17 & 0.16 & 0.32 & 93.55 & 108.7 & 391 & 199 \\
\hline Nbtree & 101 & $50.75 \%$ & 98 & $49.24 \%$ & 0.28 & 10.15 & 0.28 & 84.6551 & 96.71 & 391 & 199 \\
\hline Bagging & 76 & $38.19 \%$ & 123 & $61.80 \%$ & 0 & 0.17 & 0.29 & 98.85 & 100.02 & 391 & 199 \\
\hline Adaboostml & 82 & $41.20 \%$ & 117 & $58.79 \%$ & 0.16 & 0.168 & 0.29 & 93.10 & 97.69 & 391 & 199 \\
\hline Log Boosting & 95 & $47.73 \%$ & 104 & $52.26 \%$ & 0.23 & 0.15 & 0.301 & 83.05 & 100.44 & 391 & 199 \\
\hline
\end{tabular}

TABLE VIII. DETAILED ACCURACY By Class

\begin{tabular}{|l|l|l|l|l|l|l|}
\hline Algorithms & TP Rate & FP Rate & PRECISION & RECALL & F-MEASURE \\
\hline Navie Bayes & 0.352 & 0.159 & 0.442 & 0.352 & 0.378 \\
\hline Nbtree & 0.508 & 0.226 & 0.486 & 0.508 & 0.488 & 0.656 \\
\hline Bagging & 0.382 & 0.382 & 0.146 & 0.382 & 0.64 \\
\hline Adaboostml & 0.412 & 0.259 & 0.211 & 0.412 & 0.278 & 0.481 \\
\hline Log Boosting & 0.477 & 0.245 & 0.46 & 0.477 & 0.454 \\
\hline
\end{tabular}


Statistics accuracy by class

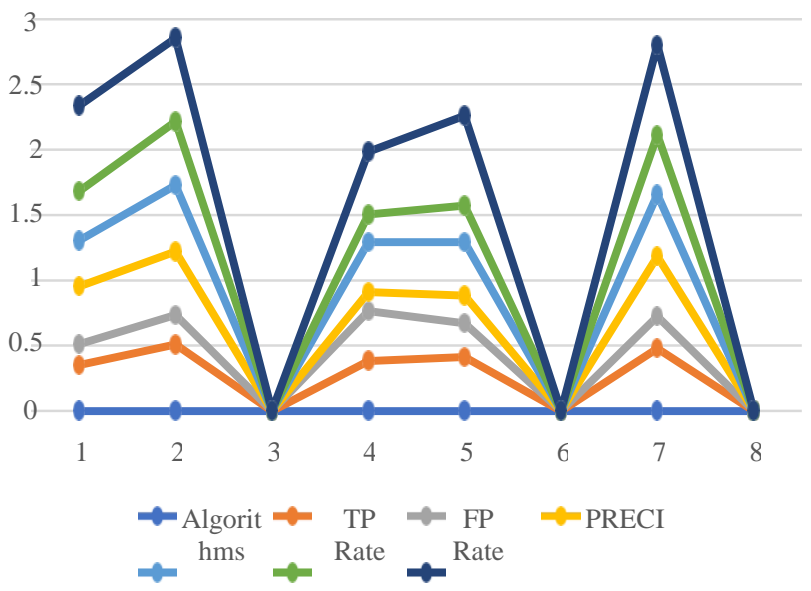

Fig. 9. Detailed Accuracy by Class.

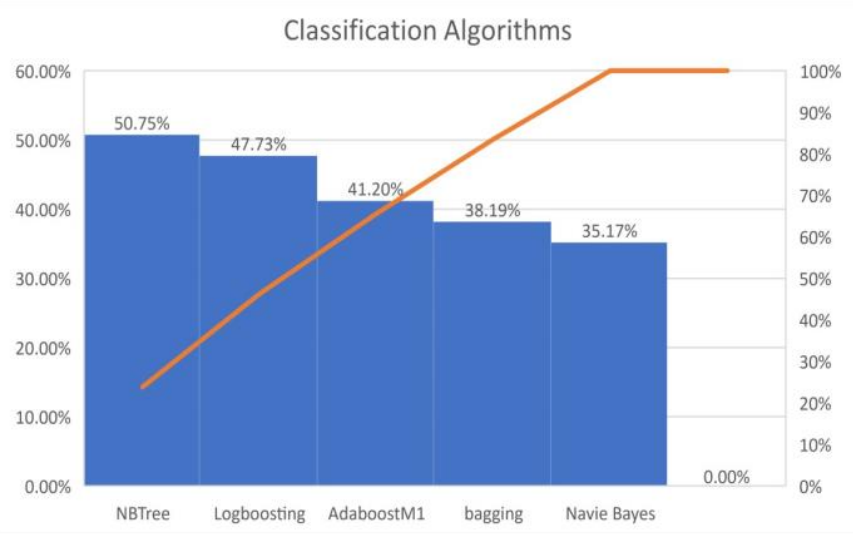

Fig. 10. Association between CCI and ICCI.

\section{Classification Techniques}

Increase Decrease

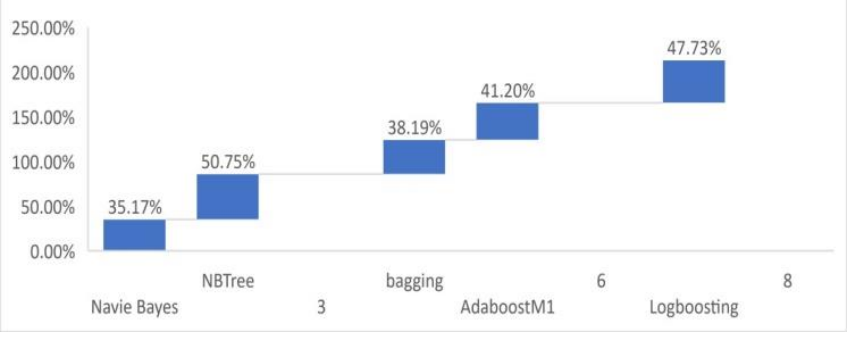

Fig. 11. Association between CCI, ICCI, ICUI, TNI.

\section{CONCLUSION}

This study looks at how to use ensemble approaches in machine learning to analyse pancreatic tumours. Researchers are working to add features like active attention and in-line memory, which will allow folding neural networks to evaluate new elements that are significantly different from what they were trained on, and preliminary results show that the proposed approach can improve the classifier's performance for early detection of pancreatic cancer. This mirrors a mammalian visual system more closely, proposing a more intelligent artificial picture recognition categorization. Even though he collects invasive samples, he increases cancer diagnosis when combined with other urine indicators in a study. Previous research has found that a panel of three protein biomarkers (LYVE1, REG1A, and TFF1) found in urine can help detect significant PDAC. We improved this panel in this study by replacing REG1A with REG1B. Finally, we will analyse four significant biomarkers that are found in urine: creatinine, LYVE1, REG1B, and TFF1. Creatinine is a protein that is commonly utilised as a kidney function indicator. Lymphatic vessel endothelial hyaluronan receptor 1 (YVLE1) is a protein that may help tumours spread. REG1B is a protein that has been linked to pancreatic regeneration, while TFF1 is trefoil factor 1, which has been linked to urinary tract regeneration and repair. This regularisation of the form's continuity allows for the smoothness of pancreatic segmentation. The preliminary result reflects the state of the art in pancreatic cancer prediction and reaches a high level of precision. However, further study is needed to detect early pancreatic cancer, because COVID-19 infection-induced pancreatic damage has gotten minimal attention. Moving further, we must compare the mood analysis of Twitter API with COVID-19 examples for pancreatic cancer detection and apply advanced innovation algorithms to existing Hadoop ecosystem work using deep learning and learning paradigms in the goal of early pancreatic cancer detection. As additional samples from various central institutions are collected and the best-performing classification model is established, preoperative diagnosis and staging from a computer using samples will be of substantial therapeutic benefit in the future.

\section{REFERENCES}

[1] H. Matsubayashi, H. Ishiwatari, K. Sasaki, K. Uesaka, and H. Ono," Detecting early pancreatic cancer: Current problems and future prospects," Gut Liver, vol. 14, no. 1, pp. 3036, Jan. 2020.

[2] Z.-Y. Wang, X.-Q. Ding, H. Zhu, R.-X. Wang, X.-R. Pan, and J.-H. Tong, "KRAS mutant allele fraction in circulating cell-free DNA correlates with clinical stage in pancreatic cancer patients," Frontiers Oncol., vol. 9,p. 1295, Nov. 2019.

[3] Morris, J. P.; Cano, D. A.; Sekine, S.; Wang, S. C.; Hebrok, M. betacatenin blocks Kras-dependent reprogramming of acini into pancreatic cancer precursor lesions in mice. J. Clin. Invest. 2010, 120, 508-520.

[4] Shamsaldin, A. S., Rashid, T. A., Al-Rashid Agha, R. A., Al-Salihi, N. K., \&Mohammadi, M. (2019). Donkey and smuggler optimization algorithm: A collaborative working approach to path finding. Journal of Computational Design and Engineering, 6(4), 562-583.

[5] S. Liu, X. Yuan, R. Hu, S. Liang, S. Feng, Y. Ai, and Y. Zhang, "Automatic pancreas segmentation via coarse location and ensemble learning," IEEE Access, vol. 8, pp. 29062914, 2020.

[6] Suram, A.; Kaplunov, J.; Patel, P. 1.; Ruan, H.; Cerutti, A.; Boccardi, V.; Fumagalli, M.; Di Micco, R.; Mirani, N.; Gurung, R. L.; Hande, M. P.; d'Adda di Fagagna, F.; Herbig, U. Gurung. Oncogeneinduced telomere dysfunction enforces cellular senescence in human cancer precursor lesions. EMBO J. 2012, 31, 2839-2851.

[7] Glicksberg BS, Miotto R, Johnson KW, Shameer K, Li L, Chen R, Dudley JT (2018) Automated disease cohort selection using word embeddings from Electronic Health Records. Pac SympBiocomput.

[8] Miotto R, Li L, Dudley JT (2016) Deep learning to predict patient future diseases from the electronic health records. European Conference on Information Retrieval.

[9] Zhen X, Chen J, Zhong Z, Hrycushko B, Zhou L, Jiang S, Albuquerque K, Gu X (2017) Deep convolutional neural network with transfer learning for rectum toxicity prediction in cervical cancer radiotherapy: a feasibility study. Institute of Physics and Engineering in Medicine Physics in Medicine \& Biology 62. 
[10] Fave, X. et al. Using pretreatment radiomics and delta-radiomics features to predict nonsmall cell lung cancer patient outcomes. Int. J. Radiat. Oncol. Biol. Phys. 7, 588 (2017).

[11] J. Saltz, R. Gupta, L. Hou, T. Kurc, P. Singh, V. Nguyen, . J. Van Arnam, Spatialorganization and molecular correlation of tumorinfiltrating lymphocytesusing deep learning on pathology images, Cell Rep. 23 (1) (2018) 181-193.
[12] Y.H. Chang, G. Thibault, O. Madin, V. Azimi, C. Meyers, B. Johnson, . J.W.Gray, Deep learning- based nucleus classification in pancreas histologicalimages, in: 2017 39th Annual International Conference of the IEEE Engineering in Medicine and Biology Society (EMBC), IEEE, 2017,pp.672-675. 